\title{
Perfil comportamental e competência social de crianças e adolescentes com distrofia muscular de Duchenne
}

\author{
Elaine Cristina Zachi \\ Anita Taub \\ Dora Fix Ventura \\ Universidade de São Paulo
}

\begin{abstract}
Resumo
A distrofia muscular de Duchenne é uma doença genética caracterizada por enfraquecimento muscular progressivo e degeneração irreversível, acompanhados por danos sensoriais e neuropsicológicos. Os objetivos do estudo consistiram em avaliar o perfil comportamental de crianças/adolescentes com DMD e a influência do prejuízo motor, da idade no início do uso de cadeira de rodas e da idade no diagnóstico. Participaram 34 pacientes e 20 controles. Os pacientes formaram dois grupos conforme o quociente de inteligência (QI). Os pais responderam ao Inventário de Comportamentos da Infância e da Adolescência. Pacientes com DMD obtiveram escores mais baixos em Atividades e Sociabilidade ( $p<0,01$; ANCOVA). Os pacientes com QI $<80$ apresentaram menores índices de Escolaridade. O prejuízo motor e as idades referentes à cadeira e ao diagnóstico correlacionaram-se com sintomas psiquiátricos/somáticos e problemas escolares. Os achados enfatizam a necessidade de programas educacionais acerca da doença como base para o desenvolvimento de estratégias de inclusão social.
\end{abstract}

Palavras-chave: distrofia muscular de Duchenne; comportamento; problemas sociais; inteligência.

\begin{abstract}
Behavior profile and social competence in children and adolescents with Duchenne muscular dystrophy. Duchenne Muscular Dystrophy is a genetic disease characterized by progressive muscle weakness and degeneration, which are accompanied by sensory and neuropsychological losses. The aims of this study were to evaluate the behavior profile of DMD children and adolescents and examine the influence of motor impairment, age at start using a wheelchair, and age at diagnosis on behavioral characteristics. Thirty-five patients and 20 controls participated. DMD patients formed two different groups according to the intelligence quotient (IQ). Participants' parents completed the Child Behavior Checklist. DMD groups scored lower on the Activities and the Social scales. Patients with QI $<80$ presented lower indices in the School scale $(p<$ 0.01, ANCOVA). Motor impairment and age at start using a wheelchair and at diagnosis correlated with psychiatric/somatic symptoms and school problems. The findings highlight the need of educational programs concerning the disease as a basis for the development of social inclusion strategies.
\end{abstract}

Keywords: Duchenne muscular dystrophy; behavior; social problems; intelligence.

$\mathrm{A}$ distrofia muscular de Duchenne (DMD) é uma doença genética recessiva causada por mutação em um único gene no cromossomo X - a distrofina (Cyrulnik \& Hinton, 2008; Emery, 1989). Também classificada como miopatia (doença muscular), a DMD compõe o conjunto das distrofias musculares progressivas, caracterizadas pela degeneração irreversível da musculatura esquelética (Zatz, 2002).

A degeneração muscular progressiva causa incapacidade física e a necessidade do uso de cadeira de rodas a partir de cerca dos 12 anos de idade (Zatz, 2002). Em grande parte dos casos, o paciente sobrevive até a segunda década de vida, com óbito decorrente de insuficiência cardíaca ou respiratória (Vainzof \& Zatz, 2003). Entretanto, o avanço das técnicas de tratamento como ventilação mecânica e o uso de corticoesteróides tem proporcionado o aumento da sobrevida dos pacientes, de modo a alcançar a terceira década de vida (Manzur \& Muntoni, 2009).

A DMD corresponde à forma mais severa e comum de distrofia muscular, com incidência aproximada de um para cada 3.500 nascimentos masculinos (Manzur \& Muntoni, 2009). Em cerca 2/3 dos indivíduos afetados o gene defeituoso é transmitido pela mãe, enquanto $1 / 3$ dos casos de DMD resulta de mutações espontâneas (D’Angelo \& Bresolin, 2006). Mais de $90 \%$ das mulheres portadoras de mutações no gene da distrofina são assintomáticas (Emery, 2001). Entretanto, parte das portadoras pode apresentar fraqueza muscular assimétrica leve à moderada, aumento da enzima creatino-quinase $(\mathrm{Ck}) \mathrm{e} /$ ou envolvimento cardíaco, geralmente subclínico, embora haja relatos de insuficiência cardíaca grave (Yiu \& Kornberg, 2008). 
Embora o comprometimento muscular seja central na DMD, é bem estabelecida na literatura a alta taxa de retardo mental dentre os indivíduos acometidos pela doença. Estudos referentes aos quocientes de inteligência (QIs) de pacientes com DMD são frequentes. Dentre amostras estudadas, as proporções de pacientes com retardo mental (definido como QI < 70) variam da ordem de 50\% (Rae et al., 1998; Rapapport et al., 1991); 49\% (Giliberto, Ferreiro, Dalamon, \& Szijan, 2004) e 20\% (Desguerre et al., 2009; Miller, Tunnecliffe, \& Douglas, 1985). Vale ressaltar que, conforme o Manual Diagnóstico e Estatístico de Transtornos Mentais 4 ${ }^{\mathrm{a}}$ Ed. (DSM-IV), um QI abaixo de 70 não é suficiente para a caracterização clínica do retardo mental, o qual também acompanha a existência de prejuízos significativos no funcionamento adaptativo e ocorrência anterior aos 18 anos de idade como critérios diagnósticos.

O perfil intelectual de pacientes com DMD apresenta distribuição normal (Cyrulnik \& Hinton, 2008) e considerável variabilidade (Cotton, Voudoris, \& Greenwood, 2001). Enquanto uma parte dos indivíduos acometidos apresenta retardo mental severo, outros têm a função intelectual preservada (Muntoni, Torelli, \& Ferlini, 2003). A média de QI desses pacientes concentra-se em torno de 80 , o que corresponde a um desvio padrão abaixo da população geral (Cotton et al., 2001). Na avaliação de crianças de três a seis anos, verificou-se atraso no desenvolvimento em todas as medidas da Escala Vineland de Comportamentos Adaptativos aplicada aos pais (Comunicação, Atividades Diárias, Socialização e Habilidades Motoras) (Cyrulnik et al., 2008).

Dentre os estudos que investigaram a possibilidade de alterações neuropsicológicas em crianças e adolescentes, foram descritos prejuízos de memória semântica (Giliberto et al. 2004; Wicksell, Kihlgren, Melin, \& Eeg-Olofsson, 2004), memória espontânea verbal e viso-espacial de curto e longo prazo, funções executivas quanto à fluência verbal e ao planejamento de ações (Wicksel et al., 2004), compreensão de convenções sociais e memória narrativa (Hinton, De Vivo, Nereo, Goldstein, \& Stern, 2000).

A DMD também é acompanhada por perdas sensoriais. Em um estudo que utilizou vários testes para avaliação da visão de cores, a proporção de pacientes com DMD com defeito no eixo verde-vermelho foi significantemente maior do que o esperado na população geral (Costa, Oliveira, Feitosa-Santana, Zatz, \& Ventura, 2007). Perdas visuais quanto ao contraste espacial, temporal e cromático foram associadas a deleções genéticas downstream ao exon 30 em crianças com DMD (Costa, Barboni, \& Ventura, 2011).

Por outro lado, estudos investigando os aspectos psiquiátricos e comportamentais associados à DMD são escassos. Mediante a aplicação de testes psicológicos projetivos, foram observados sinais de sentimentos de isolamento, auto-depreciação, marginalização e insegurança, além de sintomas de ansiedade, labilidade afetiva, depressão e preocupações hipocondríacas, especialmente nos pacientes mais velhos (Roccella, Pace, \& Gregório, 2003). A perda da marcha é o momento crítico para a manifestação de distúrbios. Em um estudo sobre a prevalência e a tipologia de distúrbios psiquiátricos em meninos com DMD de dois a 17 anos, depressão foi o diagnóstico mais prevalente nos pacientes com idades acima de nove anos (apresentaram depressão conforme os critérios do Manual Diagnóstico e Estatístico dos distúrbios mentais (DSM-III) (Fitzpatrick, Barry, \& Garvey, 1986)

Mediante a aplicação do Inventário de Comportamentos da Infância e Adolescência (Child Behavior Checklist-CBCL) com mães e pais, observou-se, dentre meninos com DMD, maior frequência de problemas sociais em relação aos dados normativos e em comparação com pacientes com paralisia cerebral. Comparados com controles não afetados, os jovens com DMD tiveram maior frequência de problemas de atenção além dos problemas sociais (Hinton, Nereo, Fee, \& Cyrulnik, 2006).

Tendo em vista a escassez de estudos a respeito dos aspectos comportamentais de jovens com distrofia muscular de Duchenne e a sua associação com fatores clínicos da doença, o presente estudo tem como objetivos avaliar o perfil comportamental de pacientes com DMD considerando-se a influência da inteligência e examinar a possibilidade de associação entre medidas comportamentais e três aspectos clínicos: a intensidade do prejuízo motor nos membros superiores, a idade no início do uso da cadeira de rodas e a idade no diagnóstico.

\section{Materiais e métodos}

Participaram do estudo 35 pacientes com distrofia muscular de Duchenne e 20 controles. Os pacientes foram recrutados na Associação Brasileira de Distrofia Muscular (ABDIM), onde são submetidos a tratamento multidisciplinar semanalmente. $\mathrm{O}$ grupo controle foi composto por alunos dos ensinos fundamental e médio da Escola de Aplicação da Faculdade de Educação da USP (FEUSP) não acometidos por distrofias musculares, com pareamento de idade em relação ao grupo de estudo. Todos os participantes eram do sexo masculino, com idades de 6 a 17 anos. Vinte e oito pacientes com DMD (80\% da amostra) faziam uso de cadeira de rodas. Os critérios de exclusão de ambos os grupos consistiram em histórico de epilepsia, trauma crânio-encefálico e doenças genéticas, com exceção da DMD no grupo de estudo.

A tabela 1 contém dados demográficos e clínicos dos participantes. Não houve diferença significante entre os grupos quanto à idade. $\mathrm{O}$ escore médio na Escala de Brooke sugere comprometimento motor mediano dos membros superiores $(2,2 \pm 1,1)$. Os pacientes com DMD receberam o diagnóstico aos 5,6 anos em média $(D P=2,2)$. Dentre os 28 pacientes que fazem uso de cadeira de rodas, seu início ocorreu aos 9,8 anos em média $(D P=1,8)$.

Os pais ou responsáveis passaram por uma entrevista semiestruturada de anamnese para coleta de dados demográficos e clínicos. Os participantes foram submetidos à avaliação de inteligência com as escalas Wechsler para crianças (WISC-III) ou para adultos (WAIS-III), conforme a idade (WISC até 16 anos e WAIS para os demais). Os pacientes com DMD cujo comprometimento motor impediu a aplicação dos testes de execução das escalas Wechsler foram examinados com o teste de Raven. Realizou-se avaliação motora dos pacientes com DMD por meio da Escala de Brooke, instrumento específico para a doença. Para avaliação comportamental, as mães ou os pais dos participantes responderam ao Questionário de Comportamentos 
Tabela 1

Dados demográficos e clínicos dos participantes

\begin{tabular}{|c|c|c|c|c|}
\hline & Grupo DMD & Grupo Controle & $F$ & \\
\hline Idade (anos) & $12,5(2,8)$ & $11,0(3,3)$ & 2,52 & 0,10 \\
\hline Escore na Escala de Brooke & $2,2(1,1)$ & - & - & - \\
\hline Idade no diagnóstico (anos) & $5,6(2,2)$ & - & - & - \\
\hline Idade na qual iniciou uso de cadeira de rodas (anos) & $9,8(1,8)$ & - & - & - \\
\hline
\end{tabular}

da Infância e da Adolescência (CBCL). Os instrumentos de avaliação são descritos a seguir:

Escalas Wechsler de inteligência. Avaliam a capacidade intelectual. No presente estudo foram administradas as versões validadas e normatizadas para uso no Brasil, tendo sido aplicada a Escala de Inteligência Wechsler para Crianças $3^{\underline{a}}$ Ed. (WISCIII) (Figueiredo, 2002) em participantes até 16 anos e a Escala de Inteligência Wechsler para Adultos (WAIS-III) (Nascimento, 2004) nos demais. As escalas são formadas por subtestes verbais e de execução (viso-espaciais), os quais medem aspectos diversos da inteligência. Os quocientes de inteligência (QIs) são medidas de desempenho compostas que resumem os resultados obtidos em conjuntos de subtestes. O QI verbal é obtido pela soma dos pontos ponderados (corrigidos por idade) dos testes verbais e de modo análogo, o QI de Execução é calculado pelos resultados de subtestes viso-espaciais. A soma dos pontos ponderados de subtestes verbais e de execução produz o QI Total, como uma medida da capacidade intelectual geral. Conforme a interpretação qualitativa dos QIs totais, valores abaixo de 70 são sugestivos de retardo mental. Entretanto, neste estudo o ponto de corte foi ajustado no valor 80 como limite de normalidade para subdividir os pacientes com DMD em 2 grupos: um com QI $<80$, o qual reúne pacientes com indício de retardo mental e com QI na faixa limítrofe e o outro grupo formado por pacientes com $\mathrm{QI} \geq 80$ que compreende a faixa de normalidade e os valores acima da média.

Teste das matrizes progressivas - escala geral. O Teste das Matrizes Progressivas de Raven- Escala Geral (Campos, 2004) pode substituuir as escalas Wechsler de inteligência para exame do potencial intelectual de pacientes com DMD, já que a dificuldade motora impossibilita uma aplicação dos subtestes de execução. A escala é formada por 60 problemas divididos em cinco séries, A, B, C, D e E, cada qual composta por 12 matrizes. Em cada matriz falta uma figura para que o examinando selecione, dentre oito possibilidades, aquela que completa a matriz formando uma sequência lógica. A resposta correta revela a capacidade de descobrir relações entre as figuras, mediante o desenvolvimento sistemático de raciocínio lógico. Os percentis gerados no teste de Raven foram convertidos em QIs, considerando-se a tabela de conversão Percentil-QI apresentada por Strauss, Sherman e Spreen (2006).

Inventário de comportamentos da infância e da adolescência $(C B C L)$. O CBCL consiste em um inventário para o exame comportamental de crianças e adolescentes de seis a 18 anos. O estudo de validação para a população brasileira baseou-se na primeira edição do instrumento, publicada em 1991 (Bordin, Mari, \& Caeiro, 1995). A segunda edição do inventário, utilizada no presente estudo, inclui 112 itens referentes a problemas de comportamento (Achenbach \& Rescorla, 2001). O inventário é respondido por um informante de convívio próximo da criança ou adolescente (mãe, pai ou responsável). O informante determina a taxa de frequência de cada comportamento em uma escala Likert de três pontos $(0=$ a afirmação não é verdadeira, $1=$ algumas vezes verdadeira, 2 = frequentemente verdadeira). O perfil social é avaliado por três Escalas de Competências Sociais: Atividades, Sociabilidade e Escolaridade. O perfil comportamental é constituído nas oito Escalas de Problemas de Comportamento: Ansiedade/Depressão, Isolamento/Depressão, Queixas Somáticas, Problemas Sociais, Problemas de Pensamento, Problemas de Atenção, Comportamento de Quebrar-Regras e Agressividade. Agrupadas, as escalas de Ansiedade/Depressão, Isolamento/Depressão e Queixas Somáticas formam a dimensão de Problemas de Introversão, enquanto as escalas de Comportamento de Quebrar-Regras e Comportamento Agressivo compõem a dimensão de Problemas de Extroversão. O inventário também contém seis escalas orientadas pelo Manual Diagnóstico e Estatístico de Transtornos Mentais (DSM-III): Problemas Afetivos, Problemas de Ansiedade, Problemas Somáticos, Problemas de Déficit de Atenção e Hiperatividade, Problemas de Comportamento Oposicional e Desafiador e Problemas de Conduta.

Brooke Upper Extremity Functional Rating Scale. A Escala de Brooke (Brooke et al., 1981) foi utilizada para a avaliação da motricidade dos membros superiores (braços e mãos). A elaboração da escala teve finalidade inicial de uso exclusivo com pacientes com DMD. Não há estudo nacional de validação da escala. O estudo norte-americano de validação e confiabilidade (Florence et al., 1984) foi desenvolvido mediante um protocolo desenhado com o objetivo de avaliar o curso natural e a eficácia do tratamento da DMD. Os escores variam de zero a cinco, conforme grau crescente de comprometimento motor dos membros superiores. A pontuação cinco corresponde à situação de dificuldade mais grave, correspondente à ausência de movimento funcional das mãos.

Os pacientes com DMD subdivididos em dois grupos, um composto por pacientes com $\mathrm{QI}<80$ e o outro formado por pacientes com $\mathrm{QI} \geq 80$, foram comparados com o grupo controle quanto aos escores do CBCL. Utilizou-se análise de variância (ANOVA) para verificação das diferenças entre grupos quanto às variáveis idade e QI. Os escores $\mathrm{T}$ obtidos no $\mathrm{CBCL}$ foram comparados por meio de análise de covariância (ANCOVA), utilizando-se os valores de QI como covariável. Verificou-se a associação entre os escores T do $\mathrm{CBCL}$ e variáveis clínicas dos pacientes com DMD (escore na Escala de Brooke, idade no diagnóstico e idade no início do uso de cadeira de rodas) 
por meio da correlação de Pearson. O nível de significância foi ajustado em $\mathrm{p}<0,05$.

O estudo é parte de um projeto maior, aprovado pelo Comitê de Ética em Pesquisa do Hospital Universitário da Universidade de São Paulo. A autorização da participação no estudo realizouse mediante leitura e assinatura de Termo de Consentimento Livre e Esclarecido pela mãe, pai ou responsável pela criança/ adolescente, anteriormente à coleta de dados.

\section{Resultados}

A tabela 2 mostra a comparação das idades e dos valores de QIs entre os três grupos distintos: pacientes com DMD e QI < 80 , pacientes com DMD e QI $\geq 80$ e controles. Dezoito pacientes com DMD (51\% da amostra inicial formada por 35 pacientes) obtiveram QI $<80$. Os 3 grupos não apresentaram diferença significante quanto à idade, mas são diferentes entre si quanto ao QI (ANOVA, $p<0,01)$.

Os escores obtidos por pacientes e controles nas escalas do CBCL, assim como os resultados das análises de covariância referentes aos mesmos, são listados na tabela 3. Na análise das Escalas de Competências Sociais do CBCL, o grupo controle obteve escores maiores com significância estatística (ANCOVA, QI como covariável, $p<0,01)$ nas escalas de Atividades e Sociabilidade e Competência Social Total. O grupo de pacientes com DMD e QI $<80$ apresentou os menores escores na escala de Escolaridade $(p<0,01)$. Por outro lado, o grupo controle revelou problemas de comportamento em comparação aos grupos com DMD, apresentando escores mais altos nas escalas de Comportamento de Quebrar-Regras, Problemas de Extroversão e de Problemas de Conduta (ANCOVA, QI como covariável, $p<$ 0,05). Entretanto, a observação dos dados normativos do CBCL mostrou que as médias de escores T obtidas pelo grupo controle nas escalas referidas (médias correspondentes a 57,4; 57,8 e 57,7

Tabela 2

Comparação entre grupos quanto as variáveis idade e QI

\begin{tabular}{|c|c|c|c|c|}
\hline & $\begin{array}{c}\text { Grupo DMD } \\
\text { QI }<80\end{array}$ & $\begin{array}{c}\text { Grupo DMD } \\
\text { QI } \geq 80\end{array}$ & $\begin{array}{c}\text { Grupo } \\
\text { Controle }\end{array}$ & \\
\hline & Média $(D P)$ & Média $(D P)$ & Média $(D P)$ & $F$ \\
\hline Idade & $12,7(2,2)$ & $12,2(3,6)$ & $11,0(3,3)$ & 1,95 \\
\hline QI & $59,0(11,4)^{*}$ & $94,1(9,6)^{*}$ & $109,4(11,9)^{*}$ & 100,5 \\
\hline
\end{tabular}

respectivamente) encontram-se dentro do limite de normalidade (escore $T$ limite $=65)$ (Achenbach \& Rescorla, 2001).

$\mathrm{Na}$ avaliação da associação entre variáveis clínicas e os escores do CBCL, o grupo de pacientes com DMD e QI $<80$ apresentou correlação positiva entre os escores da Escala de Brooke e os escores nas escalas de Ansiedade/Depressão ( $r$ $=0,61)$, Problemas do Pensamento $(r=0,67)$, Problemas de Introversão $(r=0,63)$ e Problemas Totais $(r=0,71)$, evidenciando maior sintomatologia quanto maior o comprometimento motor dos membros superiores. Por outro lado, a idade no início do uso de cadeira de rodas apresentou correlação significativa inversa com os escores de Queixas Somáticas $(r=-0,68)$, Comportamento de Quebrar-Regras $(r=-0,68)$ e Problemas Somáticos $(r=-0,76)$. O grupo com DMD e QI $\geq 80$ apresentou um perfil diferente, com correlação inversa entre os escores da Escala de Brooke e Problemas de Extroversão $(r=-0,85)$ e Problemas de Oposição e Desafio $(r=-0,82)$. Neste grupo, a idade no diagnóstico revelou correlação negativa com Escolaridade $(r=-0,76)$, Comportamento de Quebrar-Regras $(r=-0,77)$ e Comportamento Agressivo $(r=-0,76)$.

\section{Discussão}

No presente estudo, avaliou-se o perfil comportamental e competência social de pacientes com distrofia muscular de Duchenne. Verificou-se a associação entre o perfil comportamental, a idade do diagnóstico e o comprometimento motor através de informações obtidas com o cuidador principal.

$\mathrm{O}$ comprometimento global referente às Competências Sociais verificado no grupo de pacientes com QI $<80$ corrobora achados de estudos prévios acerca da deficiência intelectual, independente de outras condições médicas, nos quais crianças e adolescentes com inteligência em nível limítrofe ou retardo mental com graus variados obtiveram baixos escores nas escalas de Atividades, Sociabilidade e Escola do CBCL (Koskentausta, Iivanainen, \& Almqvist, 2004; Ruiter, Dekker, Verhulst, \& Koot, 2007).

Entretanto, na comparação com o grupo controle, o grupo com $\mathrm{QI} \geq 80$ apresentou escores rebaixados somente nas escalas de Atividades e Sociabilidade. Estas contêm itens relacionados à participação em esportes, brincadeiras, jogos, passatempos e em algum tipo de organização tal como clubes (Atividades) e ao relacionamento com familiares, amigos e outras crianças e adolescentes (Sociabilidade), de modo que a DMD afeta negativamente ambas as dimensões independentemente do nível de inteligência, uma vez que os pacientes com $\mathrm{QI}<80$ também evidenciaram problemas nestas áreas. Por outro lado, o equilíbrio entre os escores de Escolaridade (aproveitamento em disciplinas escolares) entre o grupo com QI $\geq 80$ e os controles revela a possibilidade de um bom aproveitamento escolar dentre pacientes sem problemas intelectuais, apesar das dificuldades motoras.

Estudos prévios acerca da DMD mostram limitações em atividades e na participação social em crianças e adolescentes acometidos pela doença, evidenciando associação entre tais restrições e prejuízos diversos referentes ao estado físico, a aspectos psicossociais e ao desempenho de papéis (Bray, Bundy, Ryan, North, \& Burns, 2011). A importância do suporte social para o desempenho em atividades em grupo e em redes 
Tabela 3

Comparação dos escores Tobtidos nas escalas do CBCL com QI como covariável

\begin{tabular}{|c|c|c|c|c|}
\hline & $\begin{array}{c}\text { Grupo DMD } \\
\mathrm{QI}<80\end{array}$ & $\begin{array}{c}\text { Grupo DMD } \\
\text { QI } \geq 80\end{array}$ & $\begin{array}{l}\text { Grupo } \\
\text { Controle }\end{array}$ & \\
\hline & Média $(D P)$ & Média $(D P)$ & Média $(D P)$ & $F$ \\
\hline \multicolumn{5}{|l|}{ Escalas de Competências Sociais } \\
\hline Atividades & $25,5(1,5)$ & $25,4(1,2)$ & $43,1(9,9)^{*}$ & 27,3 \\
\hline Sociabilidade & $28,7(4,8)$ & $32,8(6,7)$ & $42,5(2,8)^{*}$ & 4,3 \\
\hline Escolaridade & $30,8(9,2)^{*}$ & $43,9(7,0)$ & $44,9(8,4)$ & 12,9 \\
\hline Competência Social Total & $20,5(3,1)$ & $24,8(2,8)$ & $39,9(9,0)^{*}$ & 14,9 \\
\hline \multicolumn{5}{|l|}{ Escalas de Problemas de Comportamento } \\
\hline Ansiedade/Depressão & $60,5(7,4)$ & $56,6(6,7)$ & $60,7(7,2)$ & 1,83 \\
\hline Isolamento/Depressão & $62,5(7,5)$ & $62,0(10,9)$ & $59,3(8,0)$ & 1,84 \\
\hline Queixas Somáticas & $59,3(7,0)$ & $59,8(6,9)$ & $59,4(6,3)$ & 1,94 \\
\hline Problemas Sociais & $61,3(6,4)$ & $57,6(4,3)$ & $58,9(6,7)$ & 0,80 \\
\hline Problemas de Pensamento & $60,7(7,1)$ & $56,1(8,0)$ & $58,8(7,9)$ & 3,10 \\
\hline Problemas de Atenção & $55,0(5,9)$ & $53,0(4,4)$ & $55,7(3,4)$ & 3,00 \\
\hline Comportamento de Quebrar-Regras & $52,7(3,4)$ & $52,3(2,7)$ & $57,4(3,9)^{*}$ & 2,27 \\
\hline Comportamento Agressivo & $56,0(7,4)$ & $56,9(7,0)$ & $59,3(8,9)$ & 2,04 \\
\hline \multicolumn{5}{|c|}{ Escalas de Problemas de Introversão e de Extroversão } \\
\hline Problemas de Introversão & $61,5(8,1)$ & $59,5(8,0)$ & $60,3(4,2)$ & 1,50 \\
\hline Problemas de Extroversão & $52,8(7,3)$ & $53,6(8,9)$ & $57,8(1,6)^{*}$ & 2,76 \\
\hline Problemas Totais & $58,0(6,3)$ & $55,5(7,2)$ & $56,6(2,6)$ & 5,21 \\
\hline \multicolumn{5}{|l|}{ Escalas Orientadas pelo DSM } \\
\hline Problemas Afetivos & $58,4(7,6)$ & $60,0(7,9)$ & $60,7(8,0)$ & 3,75 \\
\hline Problemas de Ansiedade & $63,8(8,0)$ & $60,3(5,3)$ & $60,0(6,2)$ & 0,20 \\
\hline Problemas Somáticos & $56,8(3,4)$ & $56,2(6,1)$ & $58,4(7,0)$ & 0,54 \\
\hline Déficit de Atenção/Hiperatividade & $53,7(4,9)$ & $54,1(5,6)$ & $56,8(4,8)$ & 0,79 \\
\hline Problemas de Oposição e Desafio & $55,0(5,9)$ & $55,6(6,7)$ & $57,2(7,2)$ & 0,83 \\
\hline Problemas de Conduta & $53,5(4,4)$ & $52,5(3,7)$ & $57,7(6,3)^{*}$ & 2,24 \\
\hline
\end{tabular}

*ANCOVA $, p<0,01$

Nota. CBCL, Inventário de Comportamentos da Infância e da Adolescência; QI, Quociente de Inteligência;

DMD, Distrofia Muscular de Duchenne; $D P$, desvio padrão

Tabela 4

Coeficiente de correlação de Pearson para associações com significância estatística $(p<0,05)$ entre variáveis clínicas e escores T do CBCL entre os grupos com DMD

\begin{tabular}{lcc}
\hline & $\begin{array}{c}\text { Grupo DMD } \\
\text { QI }<80\end{array}$ & $\begin{array}{c}\text { Grupo DMD } \\
\text { QI } \geq 80\end{array}$ \\
\cline { 2 - 3 } & $\mathrm{r}$ & $\mathrm{r}$ \\
\hline Escala de Brooke & 0,61 & - \\
$\quad$ Ansiedade/Depressão & 0,67 & - \\
Problemas do Pensamento & 0,63 & - \\
Problemas de Introversão & - & $-0,85$ \\
Problemas de Extroversão & 0,71 & \\
Problemas Totais & - & $-0,82$ \\
Problemas de Oposição e Desafio & & \\
& & \\
Idade no início do uso de cadeira de rodas & $-0,68$ & - \\
Queixas Somáticas & $-0,68$ & - \\
Comportamento de Quebrar-regras & $-0,76$ & - \\
Problemas Somáticos & & \\
& & \\
Idade no Diagnóstico & - & $-0,76$ \\
Escolaridade & - & $-0,77$ \\
Comportamento de Quebrar-Regras & - & $-0,76$ \\
Comportamento Agressivo & &
\end{tabular}

de amizades foi ressaltada no trabalho de Fee e Hinton (2011), que mediante a utilização da escala de Competências Sociais do CBCL, verificaram a associação entre o estabelecimento de relações sociais robustas e a diminuição do risco de desenvolvimento de problemas de comportamento em crianças com DMD.
Embora haja um pressuposto de que uma condição de saúde comprometida interfira decisivamente na qualidade de vida, esta associação não é clara na literatura referente à DMD (Bray et al., 2011). Quando isolados os problemas clínicos da doença tais como o comprometimento motor e a capacidade respiratória, tanto jovens quanto adultos com DMD podem apresentar bons 
índices de qualidade de vida e ajustamento emocional, função social, relacionamento interpessoal, saúde mental e lazer, entre outros (Hendriksen et al., 2009; Kohler et al., 2005; Simon et al., 2011). Tais indicadores de qualidade de vida e ajustamento emocional podem refletir o desenvolvimento de habilidades psíquicas para enfrentamento da doença, que podem ser facilitadas pelo acesso a recursos tecnológicos tais como cadeira de rodas elétrica e telefone celular, que promovem o aumento das interações sociais e das atividades recreativas (Hendriksen et al., 2009; Kohler et al., 2005).

Utilizando a escala de Problemas Sociais pertencente ao conjunto de Escalas de Problemas de Comportamento do CBCL, Hinton et al. (2006) observaram maior proporção de pacientes com DMD com problemas sociais significativos, tanto na comparação com irmãos não afetados pela doença, quanto em relação a pacientes com paralisia cerebral. Entretanto, as alterações sociais verificadas no estudo prévio e nos nossos pacientes divergem quanto à sua natureza. Enquanto a escala de Problemas Sociais enfatiza problemas de relacionamento interpessoal (Por exemplo: 25. "Não se dá bem com outras crianças". 27. "Sente ciúme com facilidade"), as escalas de Atividades e Sociabilidade, nas quais nossos pacientes apresentaram escores rebaixados, inclui itens relacionados à frequência e à qualidade na participação em grupos de amigos, organizações e atividades esportivas ou de outra natureza. Portanto, os problemas de ordem social dos pacientes aqui avaliados remetem a uma dificuldade de acesso a situações que favoreçam a socialização.

Entretanto, contrariando as expectativas, apesar dos pacientes examinados no presente estudo apresentarem restrição de participação em atividades e grupos sociais, assim como da dificuldade de acesso a recursos tecnológicos decorrente da condição sócio-econômica, a comparação com o grupo controle não evidenciou a existência de alterações de humor, ansiedade, problemas somáticos e de atenção, alterações do pensamento ou problemas de agressividade nas escalas de Problemas de Comportamento, Problemas de Introversão/Extroversão e Escalas Orientadas pelo DSM. Este achado pode sugerir bom ajustamento emocional ou ainda, menor sensibilidade do instrumento aos fenômenos.

Nas Escalas de Problemas de Comportamento, o grupo controle apresentou maior índice de Comportamento de QuebrarRegras, o que refletiu maior sintomatologia nas escalas de Problemas de Extroversão e Problemas de Conduta, uma vez que estas escalas possuem itens em comum. Por outro lado, os resultados do grupo controle em tais escalas encontram-se dentro do limite de normalidade estabelecido no CBCL. É possível que alguns comportamentos de quebrar regras sejam esperados, em certo grau, para a faixa etária. Trata-se de itens do CBCL relativos à agressividade manifesta (por ex.: 90.“Fala palavrões”), auto-defesa (por ex.: 3.“Discute muito”) ou estratégia para obtenção de objetivos (por ex.: 43.“Mente), que podem ser inibidas em crianças com DMD, dando lugar a maior tendência à passividade e baixa assertividade. Também há que se atentar para o fato da existência de itens associados a atos motores (por ex.: 21. "Destrói objetos da sua família ou de outras crianças", 67.“Foge de casa”), os quais são limitados nos pacientes devido ao comprometimento motor dos membros superiores e do uso de cadeira de rodas.

As análises de correlação envolvendo as medidas comportamentais do CBCL e índices de comprometimento motor (escore na Escala de Brooke e idade no início do uso da cadeira de rodas) e precocidade do diagnóstico mostraram perfis distintos entre os subgrupos de pacientes com DMD divididos de acordo com o quociente de inteligência.

Os pacientes com QI $<80$ apresentaram um aumento de sintomas de ansiedade, depressão, isolamento e queixas somáticas (escalas de Ansiedade/Depressão, Problemas de Introversão e Problemas Totais), assim como de sintomas psicóticos, distúrbios do sono e problemas sexuais (Problemas de Pensamento) conforme maior comprometimento dos movimentos dos braços e das mãos, indicados pelos escores na Escala de Brooke. A susceptibilidade a alterações de humor corrobora estudos prévios que mostraram a maior incidência de sintomas de depressão em pacientes mais velhos, os quais tendem a apresentar dificuldades motoras mais proeminentes (Fitzpatrick et al., 1986; Roccella et al., 2003). A perda da marcha é apontada como o período crítico para o surgimento dos sintomas da depressão (Roccella et al. 2003). Porém, o presente estudo evidencia um agravamento dos sintomas exclusivamente em pacientes com QI $<80$, de modo a sugerir maior susceptibilidade de pacientes com inteligência com nível limítrofe ou indício de retardo mental ao desenvolvimento de alterações de humor associadas à progressão do comprometimento motor de membros superiores.

No grupo com QI $<80$, idades mais altas no início do uso da cadeira de rodas (o que implica em preservação motora dos membros inferiores por mais tempo) foram correlacionadas com menores índices de queixas/problemas somáticos e comportamento de quebrar regras. Pode-se levantar a hipótese de que o uso da cadeira proporcionou não somente uma diminuição dos problemas relacionados ao cansaço físico (item 54 da Escala de Queixas Somáticas), mas também uma redução global dos sintomas. Neste grupo, a idade no início do uso de cadeira de rodas também apresentou correlação negativa com o comportamento de quebrar regras, o que implica em uma maior agressividade manifesta e reação de transgressão de normas acompanhada da perda precoce da capacidade motora dos membros inferiores. Entretanto, deve-se atentar para o fato de que este resultado foi exclusivo de pacientes com indícios de deficiência intelectual e pode refletir uma menor habilidade de enfrentamento das dificuldades decorrentes da perda da marcha.

Por outro lado, dentre os pacientes com $\mathrm{QI} \geq 80$, o aumento do comprometimento motor dos membros superiores representado pelos escores da escala de Brooke correlacionouse com menores índices de Comportamento de Oposição e Desafio, o que refletiu menor sintomatologia na dimensão de Problemas de Extroversão. Tal achado remete às hipóteses comentadas anteriormente de uma inibição da expressão comportamental de impulsos agressivos esperados em crianças com desenvolvimento típico.

Neste mesmo grupo, idades mais altas no diagnóstico acompanharam menor participação e desempenho escolar (escala de Escolaridade), evidenciando a necessidade de um 
diagnóstico precoce que permita adaptação gradativa envolvendo a criança e a instituição de ensino, a fim de se reduzir o impacto da progressão da doença no processo de ensino. A mesma variável também mostrou correlação negativa com as escalas de Comportamento de Quebrar-Regras e de Comportamento Agressivo, o que sugere uma reação negativa ao diagnóstico acompanhada de agressividade dentre os pacientes mais novos. Tal achado ressalta a importância de intervenções precoces que visam não somente ao tratamento dos sintomas físicos, mas também dos sintomas psíquicos associados ao diagnóstico.

Em suma, as características comportamentais associadas à distrofia muscular de Duchenne revelam particularidades relacionadas ao perfil de inteligência e à progressão do comprometimento motor. A necessidade de participação social constituiu um problema comum aos pacientes com DMD e as dificuldades relacionadas ao acesso, à participação e ao desempenho na escola foram específicas dos pacientes com QI rebaixado. O caráter degenerativo e progressivo da doença pode inibir a manifestação de comportamentos agressivos que seriam esperados, em certo grau, em crianças com desenvolvimento típico. Os resultados aqui apresentados apontam para a necessidade do desenvolvimento de programas visando à educação e à sensibilização acerca da doença destinados à população e aos profissionais ligados à saúde e à escola. Tais medidas associadas ao diagnóstico precoce constituem as bases para o desenvolvimento de estratégias mais eficientes de acesso ao meio, assim como deestratégias de intervenção multidisciplinar que contemplem o perfil cognitivo e motor característico da doença.

\section{Referências}

Achenbach, T. M., \& Rescorla, L. A. (2001). Manual for the ASEBA School-Age Forms and Profiles. Burlington: University of Vermont, Research Center for Children, Youth, \& Families.

Bordin, I. A. S., Mari, J. J., \& Caeiro, M. F. (1995). Validação da versão brasileira do "Child Behavior Checklist" (CBCL) (Inventário de Comportamentos da Infância e Adolescência): dados preliminaries. Revista ABP-APAL, 17(2), 55-66.

Bray, P., Bundy, A. C., Ryan, M. M., North, K. N., \& Burns, J. (2011). Health status of boys with Duchenne muscular dystrophy: a parent's perspective. Journal of Paediatrics and Child Health, 47, 557-562. doi:10.1111/j.14401754.2011.02022.x

Brooke, M. H., Griggs, R. C., Mendell, J. R., Fenichel, G. M., Shumate, J. B. \& Pellegrino, R. J. (1981).Clinical trial in Duchenne dystrophy 1.The design of the protocol. Muscle and Nerve, 4, 186-197.

Campos, F. (2004). Teste das matrizes progressivas: escala geral séries $A, B, C$, $D$ e E. Manual. Rio de Janeiro: CEPA.

Costa, M. F., Barboni, M. S. T., \& Ventura, D. F. (2011). Psychophysical measurements of luminance and chromatic spatial and temporal contrast sensitivity in Duchenne muscular dystrophy. Psychology and Neuroscience, 4(1), 67-74. doi: 10.3922/j.psns.2011.1.008

Costa, M.F., Oliveira, A.G.F., Feitosa-Santana, C., Zatz, M., \& Ventura, D.F. (2007). Red-green color vision impairment in Duchenne muscular dystrophy. The American Journal of Human Genetics, 80, 1064-1075. doi: $10.1086 / 518127$

Cotton, S., Voudouris, N. J., \& Greenwood, K. M. (2001). Intelligence and Duchenne muscular dystrophy: full-scale, verbal, and performance intelligence quotients. Developmental Medicine and Child Neurology, 43(7), 497-501.

Cyrulnik, S. E., Fee, R. J., Batchelder, A., Kiefel, J., Goldstein, E., \& Hinton, V. (2008). Cognitive and adaptive deficits in young children with Duchenne muscular dystrophy (DMD). Journal of the International Neuropsychological Society, 14, 853-861. doi:10.10170S135561770808106X

Cyrulnik, S. E. \& Hinton, V. J. (2008). Duchenne muscular dystrophy: a cerebellar disorder? Neuroscience and Biobehavioral Reviews, 32, 486-496. doi:10.1016/j.neubiorev.2007.09.001

D’Angelo, M. G. \& Bresolin, N. (2006). Cognitive impairment in neuromuscular disorders. Muscle \& Nerve, 34,16-33. doi: 10.1002/mus.20535

Desguerre, I., Christov, C., Mayer, M., Zeller, R., Becane, H., Bastuji-Garin, S., ... \& Gherardi, R.K. (2009). Clinical heterogeneity of Duchenne muscular dystrophy (DMD): definition of sub-phenotypes and predictive criteria by long-term follow-up. Plos ONE, 4(2), 1-9. doi:10.1371/journal.pone.0004347

Emery, A. E. (1989). Clinical and molecular studies in Duchenne muscular dystrophy. Progress in Clinical \& Biological Research, 306, 15-28.

Emery, A. E. (2001). The muscular dystrophies. Nova Iorque: Oxford University Press.

Fee, R. L., \& Hinton, V. J. (2011). Resilience in children diagnosed with a chronic neuromuscular disorder. Journal of Developmental \& Behavioral Pediatrics, 32, 644-650. doi: 10.1097/DBP.0b013e318235d614

Figueiredo, V. L. M. (2002). WISC-III: escala de inteligência Wechsler para crianças: manual. São Paulo: Casa do Psicólogo.

Fitzpatrick, C., Barry, C., \& Garvey, C. (1986) Psychiatric disorder among boys with Duchenne muscular dystrophy. Developmental Medicine and Child Neurology, 28(5), 589-595. doi: 10.1111/j.1469-8749.1986.tb03900.x

Florence J. M., Pandya, S., King, W. M., Robison, J. D., Signore, L. C., Wentzell, M. \& Province, M. A. (1984). Clinical trials in Duchenne dystrophy. Standardization and reliability of evaluation procedures. Physicaltherapy, 64(1), 41-45.

Giliberto, F., Ferreiro, V., Dalamon, V., \& Szijan, I. (2004). Dystrophin deletions and cognitive impairment in Duchenne/Becker muscular dystrophy. Neurological Research, 26(1), 83-87. doi:10.1179/016164104773026589

Hendriksen, J. G. M., Poysky, J., Schrans, D. G. M., Schouten, E. G. W., Aldenkamp, A. P., \& Vles, J. S. H. (2009). Psychosocial Adjustment in males with Duchenne muscular dystrophy: psychometric properties and clinical utility of a parent-report questionnaire. Journal of Pediatric Psychology, 34(1), 69-78. doi: 10.1093/jpepsy/jsn067

Hinton, V. J., De Vivo, D. C., Fee, R. J., Goldstein, E., \& Stern, Y. (2000). Investigation of poor academic achievement in children with Duchenne muscular dystrophy. Learning Disabilities of the Research \& Practice, 19(3), 146-154. doi: 10.1111/j.1540-5826.2004.00098.x

Hinton, V. J., Nereo, N. E., Fee, R. J., \& Cyrulnik, S. E. (2006). Social behavior problems in boys with Duchenne muscular dystrophy. Developmental and Behavioral Pediatrics, 7(6), 470-476. doi: 0196-206X/06/2705-0000

Kohler, M., Clarenbach, C. F., Böni, L., Brack, T., Russi, E. W., \& Bloch, K. E. (2005). Quality of life, physical disability, and respiratory impairment in Duchenne muscular dystrophy. American Journal of Respiratory and Critical Care Medicine, 172, 1032-1036. doi: 10.1164/rccm.200503-322OC

Koskentausta, T., Iivanainen, M., \& Almqvist, F. (2004). CBCL in the assessment of psychopathology in Finnish children with intellectual disability. Research in Developmental Disabilities, 25, 341-354. doi: 10.1016/j.ridd.2003.12.001

Manzur, A. Y., \& Muntoni, F. (2009). Diagnosis and new treatments in muscular dystrophies. Journal of Neurology, Neurosurgery and Psychiatry, 80,706714. doi:10.1136/jnnp.2008.158329

Miller, G., Tunnecliffe, M., \& Douglas P. S. (1985). IQ, prognosis and Duchenne muscular dystrophy. Brain and Development., 7(1),7-9. doi.org/10.1016/ S0387-7604(85)80053-X

Muntoni, F., Torelli, S., \& Ferlini, A. (2003). Dystrophin and mutations: one gene, 
several proteins, multiple phenotypes. The Lancet Neurology, 2, 731-740. doi: 10.1016/S1474-4422(03)00585-4

Nascimento, E. (2004). WAIS-III: escala de inteligência Wechsler para adultos: manual. São Paulo: Casa do Psicólogo.

Rae, C., Scott, R. B., Thompson, C. H., Dixon, R. M., Dumughn, I., Kemp, G. J., ... \& Radda, G.K. (1998). Brain biochemistry in Duchenne muscular dystrophy: A ${ }^{1} \mathrm{H}$ magnetic resonance and neuropsychological study. Journal of the Neurological Sciences, 160, 148-157.

Rapaport, D., Passos-Bueno, M. R., Takata, R. I., Campiotto, S., Eggers, S., Vainzof, M., ... \& Zatz, M. (1992). A deletion including the brain promoter of the Duchenne muscular dystrophy gene is not associated with mental retardation. Neuromuscular Disorders, 2(2), 117-120. doi: 10.1016/09608966(92)90043-6

Roccella, M., Pace, R., \& De Gregorio, M. T. (2003). Psychopathological assessment in children affected by Duchenne de Boulogne muscular dystrophy. Minerva Pediatrica, 55(3), 267-273, 273-6.

Ruiter, K. P., Dekker, M. C., Verhulst, F. C., \& Koot, H. M. (2007). Developmental course of psychopathology in youths with and without intellectual disabilities. Journal of Child Psychology and Psychiatry, 48(5), 498-507. doi: 10.1111/j.1469-7610.2006.01712.x

Simon, V. A., Resende, M. B. D., Simon, M. A. V. P., Zanoteli, E., \& Reed, U. C. (2011). Duchenne muscular dystrophy. Quality of life among 95 patients evaluated using the life satisfaction index for adolescents. Arquivos de Neuro-Psiquiatria, 69(1), 19-22. doi: 10.1590/S0004-282X2011000100005

Strauss, E., Sherman, E., \& Spreen, O. (2006). A Compendium of neuropsychological tests. Nova Iorque: Oxford.

Vainzof, M., \& Zatz, M. (2003). Protein defects in neuromuscular diseases. Brazilian Journal of Medical and Biological Research, 36, 543-555. doi: 10.1590/S0100-879X2003000500001

Wicksell, R. K., Kihlgren, M., Melin, L., \& Eeg-Olofsson, O. (2004). Specific cognitive deficits are common in children with Duchenne muscular dystrophy. Developmental Medicine and Child Neurology, 46(3), 154-159. doi: 10.1017/ S0012162204000283

Yiu, E. \& Kornberg, A. J. (2008). Duchenne muscular dystrophy. Neurology India, 56(3), 236-247. doi: 10.4103/0028-3886.43441

Zatz, M. (2002). A biologia molecular contribuindo para a compreensão e a prevenção das doenças hereditárias. Ciência \& Saúde Coletiva, 7(1),85-99. doi: 10.1590/S1413-81232002000100008

Elaine Cristina Zachi, doutora em Psicologia pela Universidade de São Paulo, é pós-doutoranda em Psicologia Experimental na Universidade de São Paulo. Endereço para correspondência: Av. Prof. Mello Moraes, 1721, bloco A, sala D9, Butantã, cep 05508-030, São Paulo/SP. Telefone: Tel: (11) 3091-1914 / Fax: (11) 3091435. E-mail: elainez@usp.br

Anita Taub, mestre em Ciências da Saúde pela Universidade Federal de São Paulo, é psicóloga colaboradora no Instituto de Psiquiatria da Faculdade de Medicina da Universidade de São Paulo (IPq-FMUSP). E-mail: nitaub@gmail.com

Dora Fix Ventura, Livre Docente em Psicologia Experimental pelo Instituto de Psicologia da Universidade de São Paulo, é professora Titular na Universidade de São Paulo. E-mail: dventura@usp.br 